\title{
Blue-Light Transmittance In Sunglasses Over Long- Term Irradiation Within A Solar Simulator
}

\author{
Mauro Masili \\ University of São Paulo (EESC/USP) \\ Fernanda Oliveira Duarte \\ University of São Paulo (EESC/USP) \\ Liliane Ventura ( $\square$ lilianeventura@usp.br) \\ University of São Paulo (EESC/USP)
}

\section{Research Article}

Keywords: Aging test of sunglasses, Blue-light transmittance, Solar simulator, Standard ISO-12312-1, Sunglasses

Posted Date: October 12th, 2021

DOI: https://doi.org/10.21203/rs.3.rs-957061/v1

License: @ (i) This work is licensed under a Creative Commons Attribution 4.0 International License. Read Full License 


\section{ORIGINAL ARTICLE}

Title: Blue-light transmittance in sunglasses over long-term irradiation within a solar simulator

Authors: Mauro Masili, Fernanda Oliveira Duarte, Liliane Ventura*

Affiliation: Electrical Engineering Department, Engineering School of São Carlos, University of São Paulo (EESC/USP), Av. Trabalhador Sãocarlense 400, São Carlos SP, 13566-590, Brazil.

*Corresponding author: Liliane Ventura - e-mail: lilianeventura@usp.br; tel: +55 16 33739340; FAX: +55 1633739372

\# Words total: 3412

\# Words abstract: 198

\# Figures: 4

\# Tables: 1 


\begin{abstract}
Blue-light transmittance in sunglasses plays an important role for the consumer regarding eye health. Current standards do not have specific requirements for blue-light protection, as in the past. However, the literature has warned about the potential harms of blue light for the eye. The limits imposed in the past state that the average transmission in the $380 \mathrm{~nm}$ to $500 \mathrm{~nm}$ region should not exceed $1.2 \times$ the transmittance in the visible range $(380 \mathrm{~nm}-780 \mathrm{~nm})$. This work investigates, besides the transmittance of blue light in sunglasses, whether those limits imposed aiming to eye health are respected. Additionally, this study examines the blue-light transmittances preand post-artificially aging the material in a solar simulator. Twelve samples of sunglasses were tested for compliance with a former standard and submitted to the aging process up to $2500 \mathrm{~h}$ within the solar simulator. The results showed relevant changes in the lenses over time, that is, they considerably lost their blue-light attenuation capabilities. The results suggest that the aging test should be carried out on sunglasses not only for ultraviolet radiation, as required by most standards, but also for blue light. Furthermore, the standards should comprise some constraints concerning the blue-light attenuation.
\end{abstract}

Keywords Aging test of sunglasses - Blue-light transmittance - Solar simulator · Standard ISO-12312-1 $\cdot$ Sunglasses

\title{
Introduction
}

Sunglasses wearers are usually concerned with fashionable models and the protection against ultraviolet (UV) rays. The public is not aware that there are other important items and other wavelengths that are important for eye safety. Ultraviolet radiation, its harmful cumulative effects, and consequent health care are widely publicized by the strenuous work that medical societies constantly carry out to fight skin cancers. However, other potential hazardous wavelengths are on the sidelines of the public, for instance, the so-called "blue light" (380 nm $-500 \mathrm{~nm})$, which is more appropriately termed as high energy visible (HEV) light. The HEV light can be harmful to the eye if 
cumulative exposure exceeds the allowed limit, which has been established by studies carried out over the years ${ }^{1}$.

The blue-light hazard ${ }^{2}$, also known as Type II photochemically induced retinal damage $^{3}$, is associated with the absorption of the ocular system - retinal pigment epithelium and the choroid - from exposures to $380 \mathrm{~nm}$ to $520 \mathrm{~nm}$ short-wavelength light, for periods of the order of approximately $10 \mathrm{~s}$ to $1-2 \mathrm{~h}$. The retinal damage has been validated by the observed photochemical injuries in laboratory studies ${ }^{4}$.

Currently, the industry has envisioned a blue-blocking lenses market based on the possible association of short-wavelength light with maculopathies. These lenses absorb in the range of HEV light and, therefore, do not transmit blue light, failing in the Australian $^{5}$ and American coloring requirements ${ }^{6}$. They also failed in the coloring requirements of the British BS and DIN standards when they were enforced before the adoption of the European Standard EN $1836^{7}$.

The new European standard EN 12312-1, which is a mirror of ISO $12312-1^{8}$, has established the value of the relative visual attenuation quotient for traffic signal bluelight recognition, which allows the "blue blockers" certification by the old EN 1836 and current ISO 12312-1 standards. Hence, this compliance for traffic light recognition (blue, in some countries) means that these glasses do not block blue light, so the specification becomes ineffective. Dain ${ }^{1}$ has stated that it is more logical to set upper limits for blue light transmittance so that the eyes are not exposed to higher levels of HEV light than for total light transmittance. The International Commission on Nonionizing Radiation Protection (ICNIRP) also has proposed guidelines to limit the exposure to HEV light ${ }^{9}$.

As far as we know, the only effort to enforce blue-light protection in sunglasses was accomplished in the past by the British standard BS $2724^{10}$. In this standard, the average spectral transmission in the $380 \mathrm{~nm}$ to $500 \mathrm{~nm}$ region should not exceed $20 \%$ of the luminous transmittance. Although Barker supported this concept ${ }^{11}$, it was not adopted in EN $1836^{7}$.

Efforts of this group have been progressively done to have an improved eye-safe standard for sunglasses for a tropical country ${ }^{12,13,14}$ and the worldwide, as in 2013 as a member of the committee for revising the Brazilian standard ${ }^{15}$ NBR 15111:2013. In 2015, the Brazilian standard was updated to become an ISO 12312-1 mirror. Moreover, 
research in this group has been conducted to contribute to sunglasses standard requirements and to develop systems ${ }^{16,17}$ to bridge the gap of lack of instrumentation available in the industry for sunglasses certification.

This work is part of this process. It aims to investigate if unbranded sunglasses, sold in streets, meet the requirements of past standards for blue light since there is no requirement for this wavelength range in current standards. The highlight of this investigation is not only to perform the compliance test for HEV-light transmission in sunglasses but, mainly, to perform a long-term aging test aiming to verify whether there is any degradation over time of the material of the lenses, posing a potential hazard for ocular safety. As far as the authors are concerned, there are no reports in literature dealing with an aging test of sunglasses in the blue region, even for short-term periods.

\section{Methods}

Twelve random unbranded sunglasses lenses, donated by the Brazilian association of manufacturers of sunglasses (ABIÓPTICA), have been selected. The reason for selecting only 12 lenses is the constraint in the solar simulator capacity. The regular transmission spectroscopy test in all samples has been carried out, as required by the ISO 12312-1 standard ${ }^{8}$. The sunglasses had no prior information about their specifications. Out of 12 lenses, 4 are of category 4, 2 are of category 3, and 6 are of category 2. All lenses are made of synthetic materials. The chosen lenses are of those categories $(2,3$, and 4$)$ because they are the most used by sunglasses wearers.

Following the transmission spectroscopy, the lenses were submitted to the aging process as required by the ISO 12312-1 standard. The required aging process consists of artificial irradiation by a solar simulator (LEMA Xenon Suntest) with an ozone-free xenon arc lamp $(450 \mathrm{~W})$. The samples are placed $300 \mathrm{~mm}$ away from the lamp, which irradiates for $50 \mathrm{~h}$. The solar simulator is 0.46 suns, as shown in previous work ${ }^{13,14}$. To extend the required test of $50 \mathrm{~h}$, the lenses were exposed in the solar simulator in intervals of $25 \mathrm{~h}$ to complete a $2500 \mathrm{~h}$ cycle. Transmission spectroscopy was performed in all lenses to measure their luminous transmittance and their blue-light attenuation, every $25 \mathrm{~h}$ of exposure to radiation. This procedure allowed us to monitor and estimates possible ongoing deterioration of filters during the artificial aging process. The $2500 \mathrm{~h}$ 
cycle yields a radiant exposure equivalent to wearing sunglasses in a tropical country for 2 years, for 2 hours a day, according to a survey within the Brazilian population ${ }^{13}$. Therefore, this investigation is conducted on aging sunglasses consistent with these statistics.

All transmittance spectra were performed in the $200 \mathrm{~nm}-800 \mathrm{~nm}$ range, using a double-beam UV-VIS-NIR CARY 5000 (VARIAN) spectrophotometer and doublechecked with a scanning spectrophotometer from Shimadzu (UV-1800 model), both with a $1 \mathrm{~nm}$ spectral resolution. The total mean transmittance is an average of the spectral transmittance weighted by an illuminant and a proper action function.

The mean luminous transmittance, $\tau_{V}$, of the sunglasses lenses for CIE standard daylight illuminant D65 is expressed as a percentage by:

$\tau_{V}=100 \times \frac{\int_{380}^{780} \tau(\lambda) \cdot S_{D 65}(\lambda) \cdot V(\lambda) d \lambda}{\int_{380}^{780} S_{D 65}(\lambda) \cdot V(\lambda) d \lambda}$

in which $\tau(\lambda)$ is the measured spectral transmittance of the lens, $S_{D 65}(\lambda)$ is the spectral distribution of radiation of CIE standard illuminant D65, and $V(\lambda)$ is the spectral luminous efficiency for daylight (photopic) vision. Similarly, the total blue-light transmittance for sunglasses, $\tau_{B}$, is calculated in percentage as follows:

$\tau_{B}=100 \times \frac{\int_{380}^{\mathrm{bu0}} \tau(\lambda) \cdot E_{S}(\lambda) \cdot B(\lambda) d \lambda}{\int_{380}^{500} E_{S}(\lambda) \cdot B(\lambda) d \lambda}$,

in which $B(\lambda)$ is the blue-light hazard function ${ }^{8}$ and $E_{S}(\lambda)$ is the standard solar spectrum at sea level for air mass 2 (solar spectrum at ground level when the position vector of the sun is $60.11^{\circ}$ from the zenith).

According to BS 2724 standard $^{10}$, the blue-light transmittance is calculated by simple, unweighted arithmetic mean, as follows:

$\tau_{B}=100 \times \frac{\sum_{380}^{500} \tau(\lambda)}{13}$

for which the spectral resolution is taken as $10 \mathrm{~nm}$. It is noteworthy that by the period (1956) that BS 2724 standard was in force, the blue-light hazard function was still 
undiscovered and only years later (the 1970s), pioneering works ${ }^{4,18}$ raised concerns about retinal damage caused by short-wavelength visible light.

The integrals in Eqs. (1) and (2) were calculated using a 5-point Gauss-Legendre quadrature with a cubic Lagrange polynomial interpolation of the integrand.

\section{Results and discussion}

An overview of the transmittance results during the resistance to radiation test, which is an artificial aging test in a solar simulator, is presented.

Figure 1 shows an overview of the spectral transmittances of 12 selected sunglasses lenses in the visible range $(380 \mathrm{~nm}-780 \mathrm{~nm})$ before the artificial aging test in a solar simulator, that is, at $0 \mathrm{~h}$.

These lenses were submitted to a solar simulator specified by the current ISO 12312-1 standard. Figure 2 illustrates the spectral transmittances of one selected polycarbonate lens in intervals of $500 \mathrm{~h}$ of exposure in the solar simulator, up to $2500 \mathrm{~h}$. The left panel shows the luminous $(380 \mathrm{~nm}-780 \mathrm{~nm})$ visible range transmittance and the right panel shows the blue-light $(380 \mathrm{~nm}-500 \mathrm{~nm})$ transmittance.

Figure 2 demonstrates that a long-term artificial aging test degrades both the original blue and luminous transmittances, which means that the lens color is bleaching. Note that the degradation of the blue-light transmittance over time is higher than the respective luminous transmittance. Hence, the blue-light/luminous transmittance ratio increases over time, that is, as sunglasses age, the blue-light attenuation degrades faster than the bleaching effect (visible light attenuation) on the lenses. This behavior is apparent in all 12 analyzed lenses.

The time evolution of the overall blue-light attenuation, calculated by equation (2), is shown in Figure 3 for all lenses. In these samples, the blue-light hazard protection decreases as a function of the exposure period in the solar simulator. The present results for the blue light show that the rate of deterioration is greater than the corresponding deterioration rate shown in previous work regarding UV protection ${ }^{12}$. In Figure 3, trend lines (solid and dashed) are displayed for some lenses to emphasize the temporal evolution. Solid and dashed lines differentiate a slight change in the regression lines around $1000 \mathrm{~h}$ of exposition, suggesting some sort of saturation effect in the materials. 
In Figure 3, the top horizontal axis represents the equivalence between the exposure period in the solar simulator and the days of use of sunglasses in the Sun, as depicted in previous works $^{12,14}$.

Table 1 presents the results of the averaged blue-light transmittance of lenses before and after the aging test. The blue-light transmittances were computed as required by ISO 12312-1 standard [equation (2)] and by BS 2724 standard [equation (3)], respectively. The square brackets show the respective blue-light/luminous transmittance ratios. Ratios over 1.2 are marked as boldface. Before the aging test $(0 \mathrm{~h}), 4$ lenses failed the criterion of $\tau_{g} / \tau_{V}<1.2$ according to ISO standard and 6 were non-compliant as required by BS standard. After $2500 \mathrm{~h}$ exposure in the solar simulator, all lenses diminished their blue- and visible-light attenuation capabilities, that is, the average transmission of the light through the lenses increased. Table 1 shows this behavior for the blue-light transmittance. In the last 2 columns, a star highlights the specimens originally in compliance with the corresponding standard that became non-compliant at some point during the aging test. One sees that the ratio between blue and visible transmittances became non-compliant with ISO 12312-1 standard for 4 lenses and another 2 lenses did not comply with BS 2724 standard.

The current ISO aging test requires $50 \mathrm{~h}$ of exposure in a solar simulator. Note that the values in Table 1 corresponding to the 50-h test do not show evidence of any significant changes. Indeed, as pointed out in previous works ${ }^{12,14}$, the resistance to radiation test is ineffective with the parameters required by the ISO 12312-1 standard ${ }^{8}$.

As previously mentioned, at some point during the aging test, some lenses turned from compliant to non-compliant with the $1.2 \times$ criterion. Figure 4 presents the time evolution of the ratio of blue-light and visible-light transmittances, according to both ISO 121312-1 (left panel) and BS 2724 (right panel) standards.

Notice that 4 (left panel) and 2 (right panel) lenses became non-compliant if one uses the ISO $12312-1$ or BS 2724 standards, respectively, to compute the overall bluelight transmittance. In both standards, the mean visible transmittance is computed by equation (1). 


\section{Conclusion}

Current standards do not require specific parameters for eye protection against blue light. However, old superseded standards established a safe limit for blue-light overall transmittance of sunglasses, which should not exceed $20 \%$ of the total luminous transmittance. Moreover, the sunglasses blocking properties degrade over time when exposed to solar radiation. In this investigation, a set of 12 typical lenses was artificially aged in a solar simulator and blue- to visible-light transmittance ratio was determined over the aging process. The blue-light transmittances were computed under the superseded and current standards, for comparison. One found that, by the old standard, 6 lenses exceeded the $1.2 \times$ limit before the aging process and 2 became non-compliant during the test. In contrast, by the current standard, 4 lenses surpassed the suggested limit of $1.2 \times$ before the aging test and 4 lenses became non-compliant during the test. In both cases, despite the deterioration of their blocking properties over time, 4 lenses were kept in compliance with the suggested regulation. After $50 \mathrm{~h}$ of irradiation by the solar simulator, which is the current standard for the stress test, no significant changes whatsoever were observed in any of the lenses tested.

Because blue-light protection has come back to the potential eye hazard scenario, one may suggest that standards include requirements for these particular wavelengths, besides the requirement for the blue-light traffic light, and consider revising the parameters for the artificial aging process of the lenses in the solar simulator. The parameter of $2500 \mathrm{~h}$ of exposure is recommended as a period quite compatible with regular sunglasses users, or, if impractical for certification routine, a shorter period should be calculated for placing the lenses closer to the xenon arc lamp bulb.

\section{Data Availability}

The data that support the findings of this study are available from the corresponding author upon request. 


\section{References}

1. Dain, S. J. Sunglasses and sunglass standards. Clin. Exp. Optom. 86, 77-90 (2003).

2. Sliney, D. H. Optical radiation safety of ophthalmic sources. Lasers Light Ophthalmol. 8, 97-108 (1997).

3. Mellerio, J. Light effects on the retina. in Principles and Practice of Ophthalmology Light, 1326-1345 (1994).

4. Ham, W. T., Mueller, H. A. \& Sliney, D. H. Retinal sensitivity to damage from short wavelength light. Nature 260, 153-155 (1976).

5. AS/NZ-1067 Sunglasses and fashion spectacles. Reissued Inc. Amend. 1. Australian/New Zealand Standard (2009).

6. ANSI Z80.3 Nonprescription sunglasses and fashion eyewear requirements. Opt. Lab. Assoc. (2001).

7. BS EN-1836 Personal eye protection. Sunglasses and sunglare filters for general use. British Standards Institute. London (1997).

8. ISO 12312-1 Eye and face protection-sunglasses and related eyewear Part 1: sunglasses for general use. International Organization for Standardization. Geneva (2013).

9. International Commission on Non-Ionizing Radiation Protection ICNIRP guidelines on limits of exposure to incoherent visible and infrared radiation. Health Phys. 105, 7496 (2013).

10. BS-2724 Filters for protection against sun glare (for industrial and general use). British Standards Institute. London (1956).

11. Barker, F. M. Does the ANZI Z80.3 nonprescription sunglass and fashion eyewear standard go far enough? Optom. Vis. Sci. 67, 431-434 (1990). 
12. Masili, M., Duarte, F. O., White, C. C. \& Ventura, L. Degradation of sunglasses filters after long-term irradiation within solar simulator. Eng. Fail. Anal. 103, 505-516 (2019).

13. Masili, M., Schiabel, H. \& Ventura, L. Contribution to the radiation protection for sunglasses standards. Radiat. Prot. Dosimetry 164, 435-443 (2015).

14. Masili, M. \& Ventura, L. Equivalence between solar irradiance and solar simulators in aging tests of sunglasses. Biomed. Eng. Online 15, article number 86 (2016).

15. ABNT NBR-15111 Óculos para proteção solar, filtros para proteção solar para uso geral e filtros para observação direta do sol. Associação Brasileira de Normas Técnicas (2013).

16. Magri, R., Masili, M., Duarte, F. O. \& Ventura, L. Building a resistance to ignition testing device for sunglasses and analysing data: a continuing study for sunglasses standards. Biomed. Eng. Online 16, article number 114 (2017).

17. Mello, M. M., Lincoln, V. A. C. \& Ventura, L. Self-service kiosk for testing sunglasses. Biomed. Eng. Online 13, article number 45 (2014).

18. Harwerth R. S. \& Sperling H. G. Prolonged color blindness induced by intense spectral lights in rhesus monkeys. Science 174, 520-523 (1971).

\section{Acknowledgements}

This work was supported by Fundação de Amparo à Pesquisa do Estado de São Paulo FAPESP (grant number: 2013/08038-7, coordinator Mauro Masili; and 2018/16275-2, coordinator Liliane Ventura) and funded in part by Coordenação de Aperfeiçoamento de Pessoal de Nível Superior - Brazil (Capes) - Finance Code 001 (MM and FOD). 


\section{Author contributions}

M.M. and L.V. conceived the experiment(s) and analysed data; F.O.D. performed spectroscopy in the samples; MM wrote the paper; LV conducted the experiment. All authors reviewed the manuscript.

\section{Additional Information}

The authors declare that they have no conflict of interest.

\section{Figure legends}

Figure 1. Luminous $(380 \mathrm{~nm}-780 \mathrm{~nm})$ spectral transmittance of 12 sunglasses lenses before exposure in a solar simulator.

Figure 2. Luminous $(380 \mathrm{~nm}-780 \mathrm{~nm})$ and blue-light spectral transmittances $(380 \mathrm{~nm}-500 \mathrm{~nm})$ of a typical polycarbonate sunglasses lens up to $2500 \mathrm{~h}$ of exposure in a solar simulator in intervals of $500 \mathrm{~h}$.

Figure 3. Time evolution of the blue-light $(380 \mathrm{~nm}-500 \mathrm{~nm})$ protection. The vertical lines denote the time (in days of use in the sun for 2 hours), in the top axis, equivalent to 1 and 2-year exposure by sun simulator.

Figure 4. Time evolution of the blue-light and luminous transmittance ratio for all 12 lenses. The horizontal line at 1.2 shows the limit required by the standard BS $2724^{10}$. Left panel: blue-light transmittance computed as required by ISO 12312-1 standard [equation (2)]. Right panel: same calculation using BS 2724 standard [equation (3)]. 
Table 1. Blue-light transmittance before and after exposure of the lenses in the solar simulator. Blue-light transmittance is calculated as required by ISO 12312-1 standard [equation (2)] and by BS 2724 standard [equation (3)]. The square brackets show the respective blue/luminous transmittance ratios. Ratios greater than 1.2 are marked as boldface. The stars highlight the samples that complied with the corresponding standard and became non-compliant at some point during the test.

\begin{tabular}{|c|c|c|c|c|c|c|c|}
\hline \multirow{3}{*}{$\begin{array}{l}\text { Lens } \\
\text { Label }\end{array}$} & \multicolumn{3}{|c|}{ Initial (0 h) } & \multicolumn{2}{|c|}{$50 \mathrm{~h}$ in solar simulator } & \multicolumn{2}{|c|}{$2500 \mathrm{~h}$ in solar simulator } \\
\hline & \multirow{2}{*}{ Category } & \multicolumn{2}{|c|}{ Blue-light transmittance (\%) } & \multicolumn{2}{|c|}{ Blue-light transmittance (\%) } & \multicolumn{2}{|c|}{ Blue-light transmittance (\%) } \\
\hline & & ISO $12312-1$ & BS 2724 & ISO $12312-1$ & BS 2724 & ISO 12312-1 & BS 2724 \\
\hline 033 & 4 & $10.56[\mathbf{1 . 3 4}]$ & $15.65[\mathbf{1 . 9 9}]$ & $10.26[\mathbf{1 . 3 5}]$ & $15.16[\mathbf{2 . 0 0}]$ & $15.36[\mathbf{1 . 6 1}]$ & $19.25[\mathbf{2 . 0 2}]$ \\
\hline 040 & 4 & $7.01[\mathbf{1 . 2 9}]$ & $6.07[1.12]$ & $6.58[\mathbf{1 . 3 1}]$ & $5.69[1.14]$ & $12.23[\mathbf{1 . 6 6}]$ & *6.64 [1.31] \\
\hline 061 & 4 & $8.31[\mathbf{1 . 3 7}]$ & $13.21[\mathbf{2 . 1 9}]$ & $7.39[\mathbf{1 . 4 0}]$ & $11.95[\mathbf{2 . 2 7}]$ & $14.65[\mathbf{1 . 7 8}]$ & $18.78[\mathbf{2 . 2 8}]$ \\
\hline 120 & 4 & $6.59[\mathbf{1 . 4 1}]$ & $11.26[\mathbf{2 . 4 1}]$ & $6.31[\mathbf{1 . 4 4}]$ & $10.87[\mathbf{2 . 4 7}]$ & $12.32[\mathbf{1 . 7 5}]$ & $16.63[2.36]$ \\
\hline 161 & 2 & $26.24[0.98]$ & $32.04[\mathbf{1 . 2 0}]$ & 24.79 [0.99] & 30.49 [1.22] & *37.28 [1.22] & $40.83[\mathbf{1 . 3 4}]$ \\
\hline 183 & 2 & $16.47[0.52]$ & $19.86[0.63]$ & $17.60[0.54]$ & $21.02[0.64]$ & $26.47[0.70]$ & $28.68[0.76]$ \\
\hline 193 & 2 & $9.48[0.48]$ & $12.54[0.64]$ & $9.42[0.49]$ & $12.38[0.65]$ & $15.76[0.73]$ & $18.06[0.84]$ \\
\hline 195 & 3 & $19.82[1.10]$ & $26.02[\mathbf{1 . 4 5}]$ & $19.60[1.11]$ & $25.65[\mathbf{1 . 4 6}]$ & *30.63 [1.38] & $34.99[\mathbf{1 . 5 8}]$ \\
\hline 196 & 2 & $13.23[0.49]$ & $17.25[0.64]$ & $13.59[0.50]$ & $17.61[0.65]$ & $28.39[0.70]$ & $31.41[0.78]$ \\
\hline 267 & 2 & $9.23[0.31]$ & $13.73[0.46]$ & $9.50[0.32]$ & $13.98[0.47]$ & $23.22[0.57]$ & $27.76[0.68]$ \\
\hline 343 & 3 & $18.38[1.11]$ & $21.76[\mathbf{1 . 3 2}]$ & $18.49[1.13]$ & $21.85[\mathbf{1 . 3 4}]$ & *26.18 [1.28] & $28.66[\mathbf{1 . 4 0}]$ \\
\hline 346 & 2 & $21.24[1.05]$ & $23.39[1.15]$ & $22.05[1.06]$ & $24.10[1.15]$ & *28.87 [1.20] & *29.86 [1.24] \\
\hline
\end{tabular}


Figures

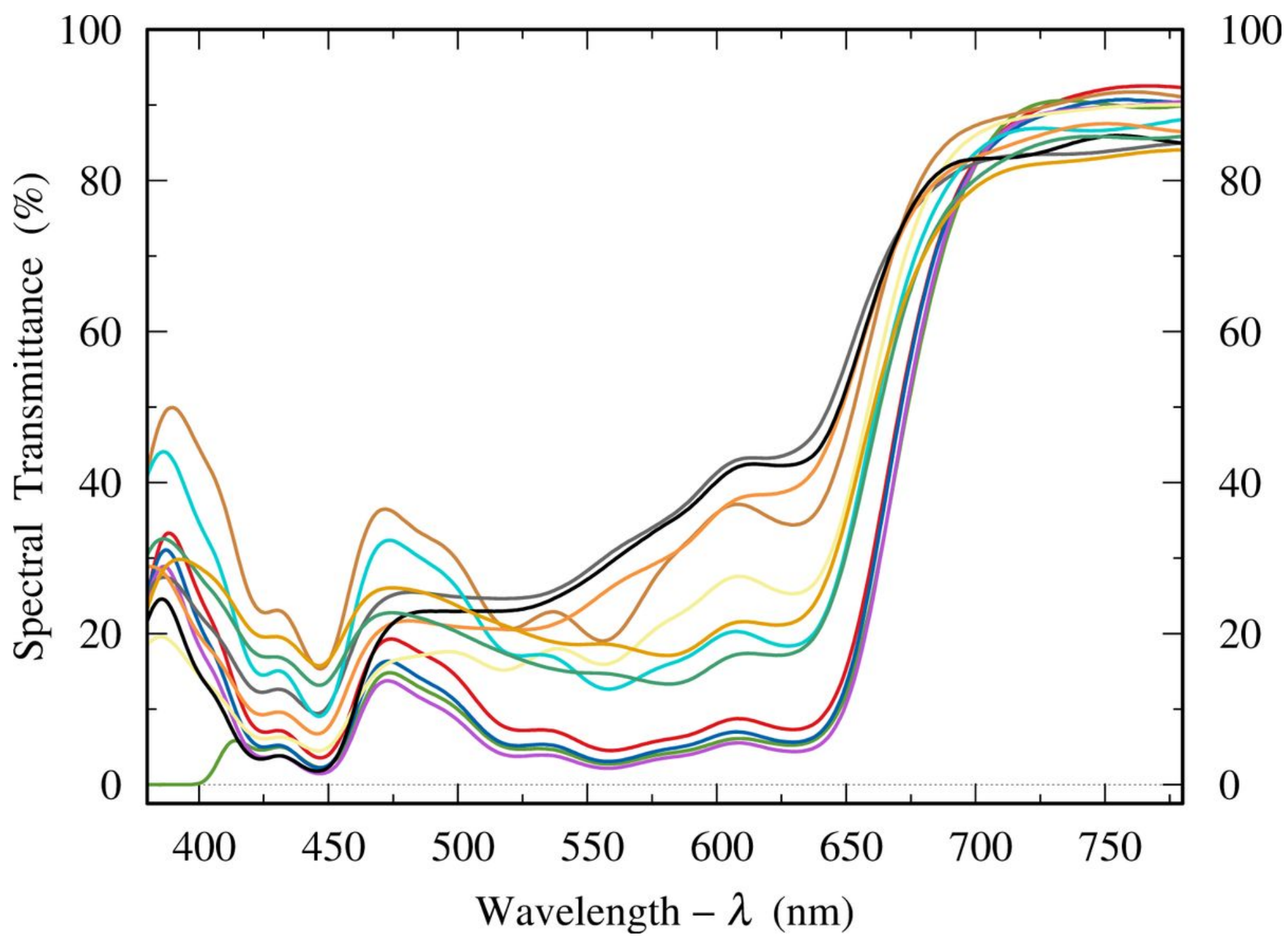

Figure 1

Luminous (380 nm - $780 \mathrm{~nm}$ ) spectral transmittance of 12 sunglasses lenses before exposure in a solar simulator. 


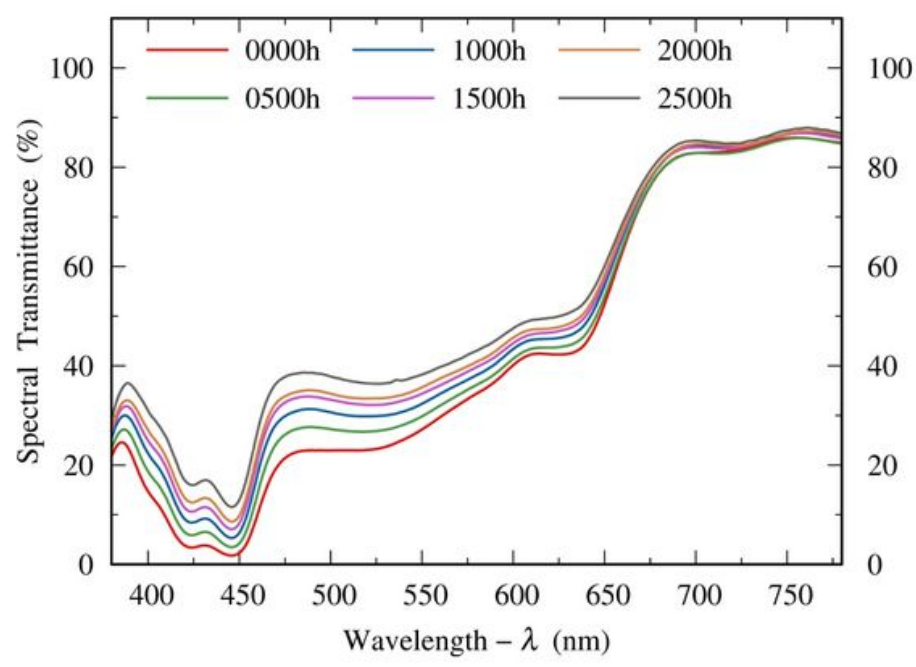

(A)

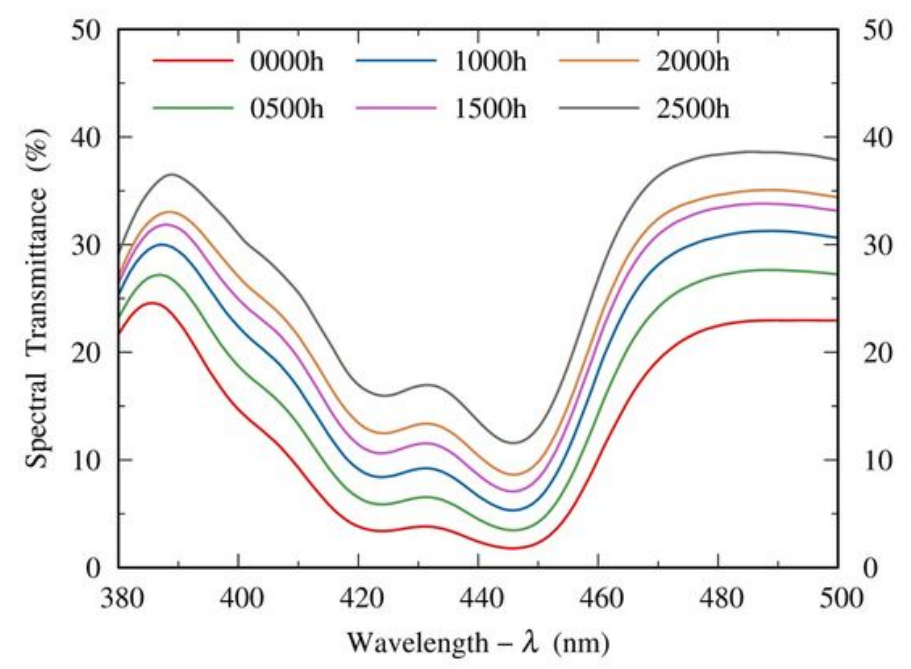

(B)

\section{Figure 2}

Luminous (380 nm - $780 \mathrm{~nm}$ ) and blue-light spectral transmittances (380 nm - 500 nm) of a typical polycarbonate sunglasses lens up to $2500 \mathrm{~h}$ of exposure in a solar simulator in intervals of $500 \mathrm{~h}$. 


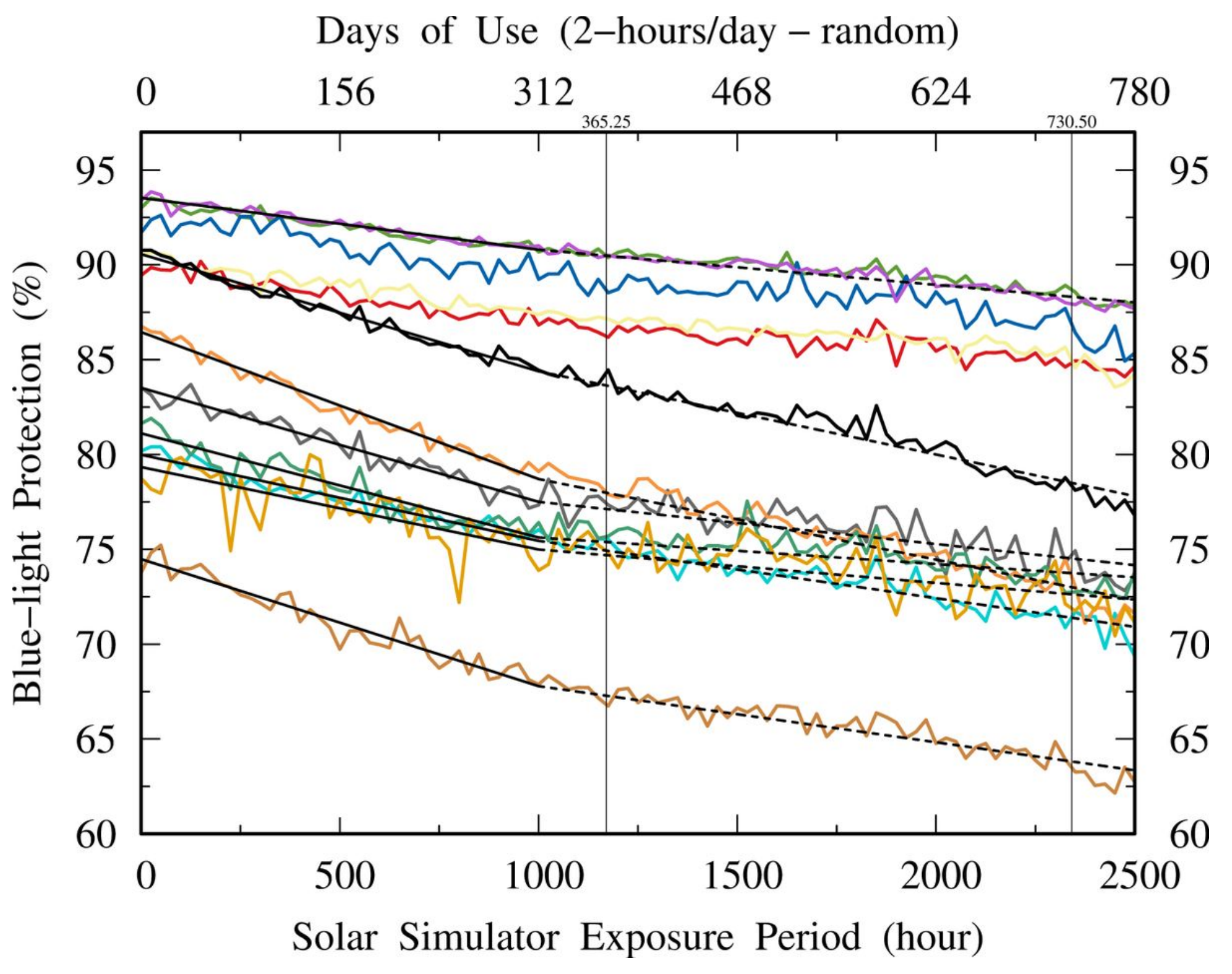

Figure 3

Time evolution of the blue-light ( $380 \mathrm{~nm}-500 \mathrm{~nm}$ ) protection. The vertical lines denote the time (in days of use in the sun for 2 hours), in the top axis, equivalent to 1 and 2-year exposure by sun simulator. 


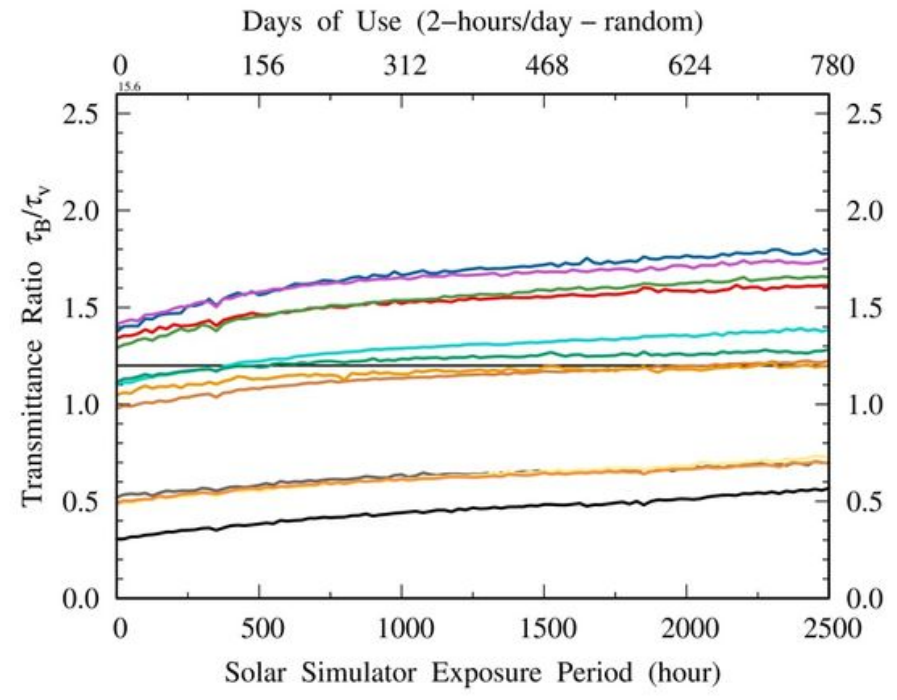

(A)

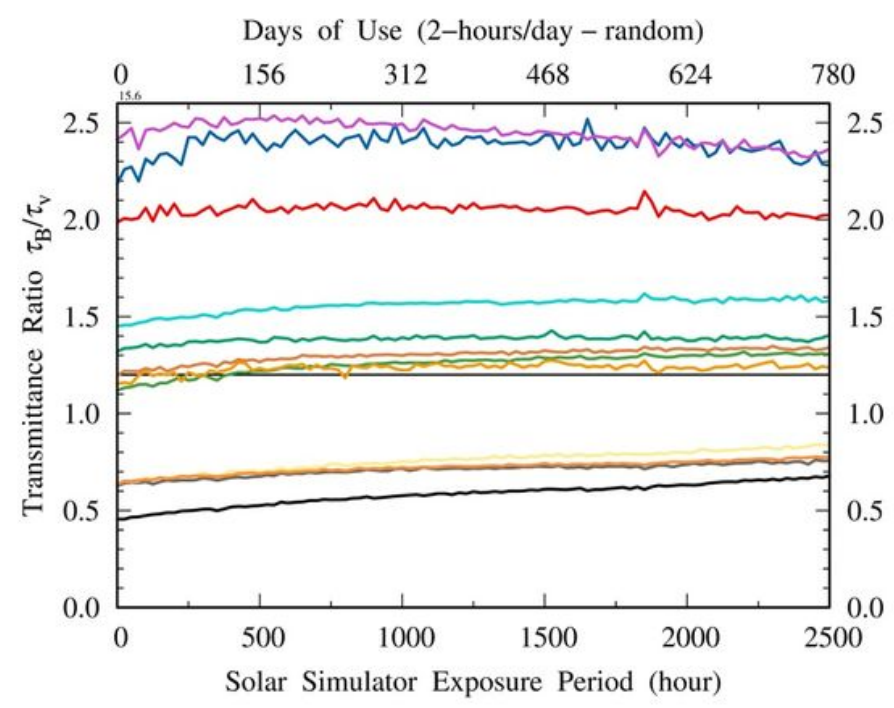

(B)

Figure 4

Time evolution of the blue-light and luminous transmittance ratio for all 12 lenses. The horizontal line at 1.2 shows the limit required by the standard BS 272410 Left panel: blue-light transmittance computed as required by ISO 12312-1 standard [equation (2)]. Right panel: same calculation using BS 2724 standard [equation (3)]. 